BULLETIN OF THE

AMERICAN MATHEMATICAL SOCIETY

Volume 77, Number 2, March 1971

\title{
AN EXISTENCE THEOREM FOR SURFACES OF CONSTANT MEAN CURVATURE
}

\author{
BY HENRY C. WENTE
}

Communicated by M. H. Protter, September 16, 1970

I. Introduction. Let $\gamma$ be an oriented, rectifiable Jordan curve in $E^{3}$ homeomorphic to the unit circle, $u^{2}+v^{2}=1$. Let $\Delta$ be the open unit disk, $u^{2}+v^{2}<1$, and let $\bar{\Delta}$ be its closure. The classical existence theorem for Plateau's problem as proven by J. Douglas [1], and T. Rado [6] asserts the existence of a minimal surface of the type of the unit disk, whose boundary is $\gamma$, and which has minimum Lebesgue area. The theorem stated in this paper is an extension of this result to surfaces of constant mean curvature.

Let $h(u, v): \bar{\Delta} \rightarrow E^{3}$ be a given minimal surface solving Plateau's problem. Let $K$ be a given constant and consider the class of continuous vector functions $\mathrm{x}: \bar{\Delta} \rightarrow E^{3}$ whose boundary values describe $\gamma$, and such that the oriented volume enclosed by $\mathrm{x}$ and $h$ is $K$. We prove that in this class there is an $x$ of minimum Lebesgue area. $x$ is a representation of a surface of constant mean curvature and satisfies the following system of equations.

(a) $\Delta x=2 H\left(x_{u} \times x_{v}\right)$

(b) $\left|x_{u}\right| \equiv\left|x_{v}\right|, \quad\left(x_{u} \cdot x_{v}\right)=0 \quad$ [conformality],

(c) $x: \partial \Delta \rightarrow E^{3}$ is an admissible representation of $\gamma$.

Previous existence theorems for the system (1) have been given by E. Heinz [2], H. Werner [8], and S. Hildebrandt [3]. They proved that if $\gamma$ is contained in the unit ball, $x^{2}+y^{2}+z^{2} \leqq 1$, and if $H$ with $|H| \leqq 1$ is given, then there exists a solution to the system (1) which is itself contained in the unit ball.

We now give a more precise statement of the theorem.

II. Statement of theorem. Denote by $s(\gamma)$ the set of vector functions $\mathrm{x}: \overline{\boldsymbol{\Delta}} \rightarrow E^{3}$ continuous on $\overline{\boldsymbol{\Delta}}$, continuously differentiable on $\boldsymbol{\Delta}$, whose boundary values are an admissible representation of the oriented Jordan curve $\gamma$, and such that the "Dirichlet" integral

$$
D(\mathrm{x}) \equiv \iint_{\mathbf{\Delta}}\left|\mathbf{x}_{u}\right|^{2}+\left|\mathbf{x}_{v}\right|^{2} d u d v
$$

AMS 1970 subject classifications. Primary 26A63, 28A75, 35A15, 49F99.

Key words and phrases. Plateau's problem, parametric surface, constant mean curvature, Dirichlet integral, oriented volume functional, Sobolev Hilbert space. 
is finite. We assume that $\delta(\gamma)$ is not empty. It is well known that this is true if $\gamma$ is rectifiable, for example.

For each $x \in S(\gamma)$ the oriented volume functional

$$
V(\mathbf{x}) \equiv(1 / 3) \iint_{\mathbf{\Delta}} \mathbf{x} \cdot\left(\mathbf{x}_{u} \times \mathbf{x}_{v}\right) d u d v
$$

is well defined and finite. Also each $x \in S(\gamma)$ is a representation of a parametric surface whose Lebesgue area does not exceed $D(x) / 2$.

Theorem 1. Let $K$ be a given constant. Let $\mathrm{s}(\gamma, K)$ denote those members of $\delta(\gamma)$ for which $V(x)=K$. There is a member of $\delta(\gamma, K)$ of minimum Lebesgue area, which is a representation of a parametric surface of constant mean curvature satisfying the system (1) for some constant $H$.

III. Indication of proof. Let $W_{1}$ be the Sobolev Hilbert space of vector valued functions $\mathrm{x}: \Delta \rightarrow E^{3}$ for which $|\mathrm{x}|,\left|\mathrm{x}_{u}\right|$, and $\left|\mathrm{x}_{v}\right|$ are square integrable. As shown by C. B. Morrey [4] each $x \in W_{1}$ has a well-defined boundary function $\mathrm{x}: \partial \Delta \rightarrow E^{3}$ which is in $L_{2}(\partial \Delta)$. Let $\Im(\gamma)$ denote those members of $W_{1}$ whose boundary values are an admissible representation of the oriented Jordan curve $\gamma$. From the results in [7] it is known that the oriented volume functional $V(x)$ on $\delta(\gamma)$ has a well-defined continuous extension to all of $\Im(\gamma)$.

THEOREM 2. Let $K$ be a given constant. Let $\Im(\gamma, K)$ be those members of $J(\gamma)$ with $V(x)=K$. There is a member of $J(\gamma, K)$ of minimum "Dirichlet" norm, $D(\mathrm{x})$.

It then follows from the results in [7], that any vector function which solves Theorem 2 also is a solution to our initial theorem.

REMARK. The results stated here do not preclude the possibility of branch points for our surface (i.e. points where $\left|x_{u}\right|=\left|x_{v}\right|=0$ ). Hildebrandt has shown that such points must be isolated in $\Delta$. Recently, R. Osserman [5] has shown that if $h(u, v): \bar{\Delta} \rightarrow E^{3}$ is a conformal representation of a minimal surface satisfying the system (1) with $H=0$ and which minimizes area, then $h$ has no branch points. It would be interesting to know whether or not the same may be said for any vector function which solves Theorem 1 .

\section{BIBLIOGRAPHY}

1. J. Douglas, Solution of the problem of Plateau, Trans. Amer. Math. Soc. 33 (1931), 263-321.

2. E. Heinz. Über die Existenz einer Fläche konstanter mittlerer Krümmung bei vorgegebener Berandung, Math Ann. 127 (1954), 258-287. MR 16,1115. 
3. S. Hildebrandt, On the Plateau problem for surfaces of constant mean curvature, Comm. Pure Appl. Math. 23 (1970), 97-114.

4. C. B. Morrey, Multiple integrals in the calculus of variations, Die Grundlehren der math. Wissenschaften, Band 130, Springer-Verlag, New York, 1966. MR 34 \#2380.

5. R. Osserman, $A$ proof of the regularity everywhere of the classical solution to Plateau's problem, Ann. of Math. (2) 91 (1970), 550-569.

6. T. Rado, On Plateau's problem, Ann. of Math. 31 (1930), 457-469.

7. $\mathrm{H}$. Wente, An existence theorem for surfaces of constant mean curvature, J. Math. Anal. Appl. 26 (1969), 318-344. MR 39 \#4788.

8. H. Werner, Problem von Douglas für Flächen konstanter mittlerer Krümmung, Math. Ann. 133 (1957), 303-319. MR 20 \#1838.

Tufts University, Medford, Massachusetts 02155 